\title{
Die deutsche Übersetzung der Distress-Intoleranz-Skala
}

\author{
Josephine Otto Michael Linden \\ Forschungsgruppe Psychosomatische Rehabilitation an der Charité Universitätsmedizin Berlin, Berlin, Deutschland
}

\section{Schlüsselwörter \\ Distress-Toleranz · Distress-Intoleranz - Psychosomatik · Fragebogen · Diagnostik · Therapieplanung}

\section{Zusammenfassung}

Distress-Intoleranz spielt eine zentrale Rolle in der Pathogenese psychischer Beschwerden. Die Verbesserung von Distress-Intoleranz ist ein häufiges Ziel, sowohl von Verhaltenstherapeuten als auch von Zuweisern oder Patienten. Deutschsprachige und vor allem ökonomische Instrumente fehlen bisher. Die neu übersetze Distress Intolerance Scale soll diese Lücke schließen. Die hier vorgestellten ersten Ergebnisse an einer psychosomatischen Stichprobe sind in Bezug auf die Item- und Skalenkennwerte vielversprechend und sollten Ausgangspunkt für weitere Untersuchungen sein.

(c) 2019 S. Karger AG, Basel

\section{The German Translation of the Distress Intolerance Scale}

\section{Keywords}

Distress tolerance - Distress intolerance - Psychosomatics . Questionnaire · Diagnostics · Treatment

\section{Abstract}

The importance of distress intolerance in psychopathology is widely known. Interventions often aim for an improvement and it is also a common goal of psychotherapists and patients. So far, appropriate questionnaires for
German-speaking professionals are lacking. In the following article we present a German translation of the Distress Intolerance Scale and first results in a psychosomatic sample. The values seem promising for using the instrument, although this should be the starting point for further research.

(c) 2019 S. Karger AG, Basel

\section{Anwendungsbereich des Fragebogens}

Erfassung von Distress-Intoleranz als subjektives Problem im Erleben und Umgang mit Stressgefühlen und Anspannungserleben für den klinischen und nicht-klinischen Bereich.

\section{Kurzbeschreibung}

Distress-Toleranz ist ein transdiagnostischer Faktor und wird definiert als die "wahrgenommene oder tatsächlich vorhandene Fähigkeit, aversive emotionale und somatische (negative Emotionen, körperliches Unwohlsein) Zustände zu tolerieren" [Leyro et al., 2010]. Es ist dabei konzeptionell von einer Dimension mit DistressToleranz an einem Pol und Distress-Intoleranz am anderen Pol auszugehen.

Die Bedeutung in der Entstehung und Aufrechterhaltung psychischer Störungen wurde durch eine Vielzahl von Untersuchungen belegt. Das Konstrukt steht unter anderem im Zusammenhang mit Angsterkrankungen, depressiven Störungen und Suchterkrankungen [Anestis et al., 2011; Asmundson et al., 2011; Clen et al., 2011; 
Schmidt et al., 2011; Vujanovic et al., 2011; Allan et al., 2014]. Erklärt wird dies mit dem Mechanismus, dass Personen mit einer hohen Distress-Intoleranz eher dysfunktionale Belastungsbewältigungsstrategien wie Vermeidungs-, Schon- oder Fluchtverhalten wählen. Personen mit einer hohen Distress-Toleranz benutzen eher adaptive Regulationsmechanismen, was zu korrigierenden Erfahrungen in der Auseinandersetzung mit Stressoren führt und eine Symptommanifestation und -chronifizierung verhindert [Jeffries et al., 2016]. Dies ist für ambulant und stationär arbeitende Verhaltenstherapeuten von besonderer Relevanz, da eine Verbesserung von DistressToleranz mit einer Verbesserung der Symptomschwere und psychosozialen Funktionsfähigkeit einhergeht [McHugh et al., 2014].

Konzeptionell wurde von Zvolensky et al. [2011] ein hierarchisches Modell mit einem globalen Distress-Toleranz-Faktor beschrieben, was von Bardeen et al. [2013] in einer konfirmatorischen Faktorenanalyse bestätigt werden konnte. Der globale Distress-Toleranz-Faktor besteht aus den fünf domänenspezifischen Faktoren Ambiguitätstoleranz, Unsicherheitstoleranz, Diskomforttoleranz, Toleranz negativer Emotionen und Frustrationstoleranz.

Es finden sich seit den 1950er Jahren in der Literatur eine Reihe von Instrumenten, um einzelne Dimensionen von Distress-Toleranz oder auch das Konstrukt ganzheitlich zu erfassen [für einen Überblick: Zvolensky et al., 2011], wobei eine Vielzahl von verhaltensbasierten Messmethoden existieren. Am populärsten ist die Exposition zu aversiven Reizen (wie Hitze- oder Kältereizen) mit der Messung der Zeit von der Äußerung negativer Befindlichkeit bis zum Abbruch des Testes. Die Differenz wird dann als Ausmaß der Distress-Toleranz gedeutet [siehe auch McHugh et al., 2011].

Im wissenschaftlichen Diskurs zeigt sich ein Dualismus zwischen verhaltensbasierten und selbstauskunftsbasierten Messinstrumenten, mit zum Teil nur geringen Korrelationen [Glassman et al., 2016]. Eine Erfassung nur einzelner Domänen von Distress (In)Toleranz führt dabei zu einer Reduktion des Zusammenhangs mit verhaltensbezogenen Kennwerten [McHugh et al., 2011; Ameral et al., 2014]. Daher entwickelten McHugh und Otto [2012] aus einem umfassenden Item- und Instrumentenpool mittels konfirmatorischer Faktorenanalyse ein Kurzinstrument mit 10 Items - die DistressIntoleranz-Skala. Diese domänenübergreifende Skala besitzt einen höheren Zusammenhang zu verhaltensbasierten Maßen. Aufgrund ihrer Kürze bietet sich die Skala ebenfalls als Messinstrument im klinischen Setting an. Im Folgenden wird eine deutsche Übersetzung vorgelegt, was den Einstieg in die wissenschaftliche wie klinische Verwendung in unserem Sprachraum bieten soll.

\section{Entwicklung des Messinstrumentes}

Die von McHugh und Otto [2012] veröffentlichte englische Kurzskala zur Erfassung von Distress-Intoleranz wurde ins Deutsche übersetzt und dann von einer unabhängigen englischsprachigen Person wieder ins Englische rückübersetzt. Es zeigten sich keine relevanten Unterschiede. Die Skala wurde im Anschluss in einer psychosomatischen Rehaklinik zum Behandlungsbeginn von Patienten ausgefüllt, wobei sich keine Hinweise auf Verständnisprobleme zeigten.

\section{Durchführung}

Die Probanden werden gebeten, das eigene Verhalten und Einstellungen im Umgang mit Belastungen allgemein abzubilden, wobei die Items der Subskalen abwechselnd präsentiert werden. Die Antwort erfolgt auf einer angepassten fünfstufigen Likert-Skala von " $1=$ stimme gar nicht zu" bis " 5 = stimme voll und ganz zu". Das Ausfüllen dauert ca. 10 Minuten.

\section{Auswertung}

Bei der Auswertung werden jeweils die Einzelitems und die Gesamtskala betrachtet. Alle Items gehen gleichwertig in die Analyse ein, und ein Umkodieren ist aufgrund einer einheitlichen Polung nicht notwendig. Es wird ein Mittelwert über alle Items als Gesamtwert berechnet.

\section{Kennwerte der deutschen Übersetzung an einer Stichrobe psychosomatischer Patienten}

Die deutsche Fassung der Distress-Intoleranz-Skala wurde von 269 Patienten einer psychosomatischen Rehabilitationsklinik ausgefüllt. Diese waren im Durchschnitt 50,81 Jahre alt (SD: 9,65), 62,6\% waren Frauen und 36,9\% besaßen Abitur als höchsten Bildungsabschluss. Die Hauptentlassungsdiagnosen waren affektive Störungen $(43,4 \%)$ gefolgt von Belastungs- und somatoformen Störungen $(33,2 \%)$.

Die Mittelwerte der einzelnen Items schwanken zwischen 3,12 und 4,08 (SD: zwischen 0,98 und 1,25). Dabei fällt das Item 6 ("Ich würde alles dafür tun, mich weniger gestresst und aufgebracht zu fühlen") durch den ausreißenden Mittelwert von 4,08 auf. Alle anderen Mittelwerte sind unter 4,00. Alle Items weisen eine negative Schiefe, das bedeutet eine linksschiefe bzw. rechtssteile Verteilung, auf. Die Gesamtskala besitzt einen Mittelwert von 3,51 (SD: 0,75). Eine Übersicht über alle Kennwerte, auch auf Itemebene, findet sich in Tabelle 1. 
Tabelle 1. Überblick über die Itemformulierungen, Itemkennwerte und Gesamtskala

\begin{tabular}{|c|c|c|c|c|c|c|c|}
\hline Nr. & Item & $\begin{array}{l}\text { Mittelwert } \\
\text { (Range) }\end{array}$ & SD & Schiefe & $\begin{array}{l}\text { Schwierig- } \\
\text { keitsindex } P\end{array}$ & $\begin{array}{l}\text { Item- } \\
\text { Trenn- } \\
\text { schärfe }\end{array}$ & $\begin{array}{l}\text { Cronbachs a } \\
\text { wenn Item } \\
\text { entfernt }\end{array}$ \\
\hline DI 1 & Es erschreckt mich, wenn ich nervös bin & $3,12(1-5)$ & 1,15 & $-0,81$ & 62,4 & 0,52 & 0,87 \\
\hline DI 2 & $\begin{array}{l}\text { Ich kann nicht damit umgehen, mich gestresst oder } \\
\text { aufgebracht zu fühlen }\end{array}$ & $3,50(1-5)$ & 1,12 & $-0,68$ & 70,0 & 0,66 & 0,86 \\
\hline DI 3 & $\begin{array}{l}\text { Andere Menschen scheinen viel besser damit umgehen zu } \\
\text { können, sich gestresst oder aufgebracht zu fühlen }\end{array}$ & $3,65(1-5)$ & 1,14 & $-0,31$ & 73,0 & 0,52 & 0,87 \\
\hline DI 4 & $\begin{array}{l}\text { Mich gestresst oder aufgebracht zu fühlen, ist eine Tortur } \\
\text { für mich }\end{array}$ & $3,78(1-5)$ & 1,10 & $-0,66$ & 75,6 & 0,66 & 0,86 \\
\hline DI 5 & Gefühle von Stress oder Aufgebrachtsein beunruhigen mich & $3,82(1-5)$ & 1,18 & $-0,33$ & 76,4 & 0,68 & 0,86 \\
\hline DI 6 & $\begin{array}{l}\text { Ich würde alles dafür tun, mich weniger gestresst und } \\
\text { aufgebracht zu fühlen }\end{array}$ & $4,08(1-5)$ & 0,98 & $-0,20$ & 81,6 & 0,40 & 0,88 \\
\hline DI 7 & $\begin{array}{l}\text { Wenn ich mich gestresst oder aufgebracht fühle, kann ich } \\
\text { nicht aufhören darüber nachzudenken, wie schlecht sich } \\
\text { dieser Zustand gerade anfühlt }\end{array}$ & $3,49(1-5)$ & 1,25 & $-0,88$ & 69,8 & 0,67 & 0,86 \\
\hline DI 8 & $\begin{array}{l}\text { Ich muss störende Gefühle sofort loswerden; ich ertrage es } \\
\text { nicht, wenn diese andauern }\end{array}$ & $3,29(1-5)$ & 1,13 & $-0,62$ & 65,8 & 0,47 & 0,87 \\
\hline DI 9 & $\begin{array}{l}\text { Ich halte Situationen, in denen ich mich aufgeregt fühlen } \\
\text { könnte, nicht aus }\end{array}$ & $3,08(1-5)$ & 1,21 & $-0,93$ & 61,6 & 0,71 & 0,86 \\
\hline \multirow[t]{2}{*}{ DI 10} & Ich kann störende Gefühle nicht aushalten & $3,38(1-5)$ & 1,13 & $-0,57$ & 67,6 & 0,69 & 0,86 \\
\hline & Gesamtskala & $3,51(1,30-5,00)$ & 0,79 & $-0,15$ & Cronbachs $\alpha$ : & & 0,88 \\
\hline
\end{tabular}

Betrachtet man die nach Kelava und Moosbrugger [2008] berechneten Schwierigkeitsindizes, finden sich Werte von 0,60 bis unter 0,80 . Eine Ausnahme bildet wieder das sechste Item der Skala mit einer vergleichsweise hohen Zustimmungsrate von $p=0,81$. Bei einem potenziellen Range von 0 bis 100 sind mittlere Schwierigkeitsindizes zu präferieren, da diese die höchste Differenzierungsfähigkeit aufweisen. Für die vorliegende Skala kann demnach über die Werte diskutiert werden (siehe Abschnitt unten). Die Itemtrennschärfen bewegen sich durchweg im positiven Bereich zwischen 0,40 und 0,71. Hier fällt das Item 6 mit einer deutlich reduzierten Trennschärfe (von 0,40) auf, Item 8 ("Ich muss störende Gefühle sofort loswerden; ich ertrage es nicht, wenn diese andauern") besitzt eine ebenfalls geringe Trennschärfe von 0,47. Eine direkte Auflistung findet sich ebenfalls in Tabelle 1.

Cronbachs $\alpha$ als Maß für interne Konsistenz und Reliabilitätsschätzer beträgt für die Gesamtskala 0,88 und ist damit sehr zufriedenstellend [Cortina, 1993; Schermelleh-Engel und Werner, 2008]. Zur Überprüfung der von den Autoren der Originalskala postulierten und von uns ebenfalls angenommenen Eindimensionalität wurde eine Hauptkomponentenanalyse durchgeführt. Bei dem Extraktionskriterium "Eigenwert $>1$ " konnte ein Faktor mit einer Gesamtvarianzaufklärung von $47,89 \%$ extrahiert werden. Nach dem Screeplot-Kriterium ergibt sich dasselbe Resultat. Sowohl der Reliabilitätsschätzer als auch das Ergebnis der explorativen Faktorenanalyse sprechen demnach für die Eindimensionalität der Skala.

Als Kennwerte der Validität der Skala wurde der Zusammenhang zur Skala "Gesamtsymptombelastung" (Global Severity Index) des SCL-90 berechnet. Es zeigte sich ein signifikant positiver Zusammenhang $(r=0,45$, $p \leq 0,001)$, was bedeutet, dass eine höhere Distress-Intoleranz mit einer höheren Symptombelastung einhergeht. Es ergaben sich keine statistisch bedeutsamen Geschlechtsunterschiede (Mittelwert Männer 3,51, SD 0,78; Mittelwert Frauen 3,51, SD 0,80; $\left.\mathrm{t}_{(257)}=0,33, p=0,974\right)$, ebenso keine statistisch bedeutsame Korrelation zum Alter des Patienten $(r=-0,03, p=0,649)$. Es fanden sich keine signifikanten Unterschiede zwischen den Gruppen mit verschiedenem Bildungsabschluss $\left(F_{(4,254)}=1,93, p=\right.$ $0,106)$.

\section{Interpretation der Ergebnisse}

Im Großen und Ganzen sind die Ergebnisse der vorliegenden Stichprobe vielversprechend.

Auf Itemebene fällt das Item 6 durch einen hohen Mittelwert und eine hohe Zustimmungsrate auf. Ebenfalls ist die Trennschärfe hier reduziert. Womöglich handelt es sich hierbei um einen Deckeneffekt, da von einer generell 
hohen Zustimmung zu diesem Item auszugehen sein dürfe und ein geringeres Stresserleben ein generell attraktives Ziel ist. Es sollte in folgenden Untersuchungen besonders im Fokus stehen, und gegebenenfalls sollten parallele Erhebungen mit und ohne dieses Item vorgenommen werden. Alle Items sind rechtssteil verteilt und besitzen vergleichsweise hohe Mittelwerte. Dies wurde in der Tendenz so erwartet und kann beispielsweise auf die klinische Stichprobe zurückgeführt werden, da belastete Patienten eine erhöhte Distress-Intoleranz aufweisen, wie im Einleitungsteil dargestellt wurde.

\section{Bisherige Erfahrungen und Ausblick}

Das Verfahren wird in der Basiserhebung der Eingangsbefragung in einer psychosomatischen Rehabilitationsklinik verwendet und im Rahmen einer wissenschaftlichen Studie als Outcome-Score genutzt. In jedem Fall sollten weitere Untersuchungen folgen: Wichtig wäre eine Überprüfung der Faktorenstruktur mittels einer konfirmatorischen Faktorenanalyse und umfangreicheren Validitätsuntersuchungen (zum Beispiel auch Krite- riumsvalidität an verhaltensbezogener Distress-Intoleranz) an einer neuen Stichprobe sowie die Erhebung an einer nicht-klinischen Stichprobe zur Herleitung von Normwerten. Eine praktische Verwendung, zum Beispiel in Form einer Therapiezielkonkretisierung, ist ebenfalls denkbar und kann eine sinnvolle Ergänzung zu symptomspezifischen Messinstrumenten darstellen. Für eine individuelle Diagnostik sollten allerdings weitere Untersuchungen (auch mit der Betrachtung von Normwerten) und eine weitere Optimierung der Items erfolgen.

\section{Statement of Ethics}

Die teilnehmenden Patienten haben ihre schriftliche Zustimmung zur Datenerhebung gegeben und wurden im Vorfeld über Sinn und Zweck der Untersuchung informiert. Das Studienprotokoll wurde vom internen Reviewboard der deutschen Rentenversicherung Bund im Hinblick auf ethische Richtlinien überprüft.

\section{Disclosure Statement}

Es bestehen keine Interessenskonflikte.

\section{Literatur}

Allan NP, Macatee RJ, Norr AM, Schmidt NB. Direct and Interactive Effects of Distress Tolerance and Anxiety Sensitivity on Generalized Anxiety and Depression. Cognit Ther Res. 2014;38(5):530-40.

Ameral V, Palm Reed KM, Cameron A, Armstrong JL. What Are Measures of Distress Tolerance Really Capturing? A Mixed Methods Analysis. Psychol Conscious (Wash D C). 2014;1:357-69.

Anestis MD, Fink EL, Smith AR, Selby EA, Joiner TE. Eating disorders. In: Zvolensky M, Bernstein A, Vujanovic A, editors. Distress tolerance. Theory, research, and clinical applications. New York: The Guilford Press; 2011.

Asmundson GJ, Peluso DL, Carleton RN, Collimore KC, Welch PG. Chronic Musculoskeletal Pain and Related Health Conditions. In: Zvolensky M, Bernstein A, Vujanovic A, editors. Distress tolerance. Theory, research, and clinical applications. New York: The Guilford Press; 2011.

Bardeen JR, Fergus TA, Orcutt HA. Testing a Hierarchical Model of Distress Tolerance. J Psychopathol Behav Assess. 2013;35(4):495-505.

Clen SL, Mennin DS, Fresco DM. Major depressive disorder. In: Zvolensky M, Bernstein A, Vujanovic A, editors. Distress tolerance. Theory, research, and clinical applications. New York: The Guilford Press; 2011.
Cortina JM. What is Coefficient Alpha? Examination of Theory and Applications. J Appl Psychol. 1993;78(1):98-104.

Glassman LH, Martin LM, Bradley LE, Ibrahim A, Goldstein SP, Forman EM, et al. A Brief Report on the Assessment of Distress Tolerance: Are We Measuring the Same Construct? J Ration Emot Cogn Behav Ther. 2016;34(2):8799.

Jeffries ER, McLeish AC, Kraemer KM, Avallone KM, Fleming JB. The Role of Distress Tolerance in the Use of Specific Emotion Regulation Strategies. Behav Modif. 2016 May;40(3): 439-51.

Kelava A, Moosbrugger H. Deskriptivstatistische Evaluation von Items (Itemanalyse) und Testwertverteilungen. In: Moosbrugger $\mathrm{H}$, Kelava A, editors. Testtheorie und Fragebogenkonstruktion. Berlin, Heidelberg: Springer; 2008.

Leyro TM, Zvolensky MJ, Bernstein A. Distress tolerance and psychopathological symptoms and disorders: a review of the empirical literature among adults. Psychol Bull. 2010 Jul; 136(4):576-600.

McHugh RK, Daughters SB, Lejuez CW, Murray HW, Hearon BA, Gorka SM, et al. Shared Variance among Self-Report and Behavioral Measures of Distress Intolerance. Cognit Ther Res. 2011 Jun;35(3):266-75.
McHugh RK, Kertz SJ, Weiss RB, Baskin-Sommers AR, Hearon BA, Björgvinsson T. Changes in distress intolerance and treatment outcome in a partial hospital setting. Behav Ther. 2014 Mar;45(2):232-40.

McHugh RK, Otto MW. Refining the measurement of distress intolerance. Behav Ther. 2012 Sep;43(3):641-51.

Schermelleh-Engel K, Werner C. Methoden der Reliabilitätsbestimmung. In: Moosbrugger $\mathrm{H}$, Kelava A, editors. Testtheorie und Fragebogenkonstruktion. Berlin, Heidelberg: Springer; 2008.

Schmidt NB, Mitchell M, Keough M, Riccardi C. Anxiety and its disorders. In: Zvolensky M, Bernstein A, Vujanovic A, editors. Distress tolerance. Theory, research, and clinical applications. New York: The Guilford Press; 2011.

Vujanovic AA, Bernstein A, Litz BT. Traumatic stress. In: Zvolensky M, Bernstein A, Vujanovic A, editors. Distress tolerance. Theory, research, and clinical applications. New York: The Guilford Press; 2011.

Zvolensky MJ, Leyro TM, Bernstein A, Vujanovic A. Historical perspectives, theory, and measurement of distress tolerance. In: Zvolensky M, Bernstein A, Vujanovic A, editors. Distress tolerance. Theory, research, and clinical applications. New York: The Guilford Press; 2011. 